\title{
Age-specific associations between systolic blood pressure and cardiovascular mortality
}

\author{
Mi-Hyang Jung, ${ }^{\oplus 1}$ Sang-Wook $\mathrm{Yi}_{,}{ }^{\circ}{ }^{2}$ Sang Joon $\mathrm{An}_{1}{ }^{3}$ Jee-Jeon $\mathrm{Yi}^{4}$
}

${ }^{1}$ Cardiovascular Center, Chuncheon Sacred Heart Hospital, Chuncheon, The Republic of Korea ${ }^{2}$ Department of Preventive Medicine and Public Health, Catholic Kwandong University College of Medicine, Gangneung, Gangwon-do, The Republic of Korea

${ }^{3}$ Department of Neurology, Catholic Kwandong University International Saint Mary's Hospital, Incheon, The Republic of Korea

${ }^{4}$ Institute of Epidemiology and Public Health, Catholic Kwandong University, Gangneung, Gangwon-do, The Republic of Korea

Correspondence to Professor Sang-Wook Yi, Department of Preventive Medicine and Public Health, Catholic Kwandong University

College of Medicine, Gangneung 25601, The Republic of Korea; flyhighysw@ gmail.com

Received 2 January 2019 Revised 4 April 2019 Accepted 8 April 2019 Published Online First 4 May 2019

\begin{abstract}
Objective We aimed to identify the following in all age groups among individuals without known hypertension and CVD: (1) Whether a systolic blood pressure (SBP) of 130-139 mm Hg elevates cardiovascular disease (CVD) mortality. (2) Whether SBP shows a linear association with cause-specific CVD mortality.

Methods We used the Korean National Health Insurance sample data ( $n=429220)$. Participants were categorised into three groups by age $(40-59$ years, 60-69 years and $70-80$ years).

Results During 10.4 years of follow-up, 4319 cardiovascular deaths occurred. A positive and graded association was generally observed between SBP and overall and cause-specific CVD mortality regardless of age, except for ischaemic heart disease (IHD) mortality in those aged $70-80$ years. Among those aged 70-80 years, the HRs (95\% Cls) for overall CVD mortality were 1.08 (0.92-1.28), 1.14 (0.97-1.34) and 1.34 (1.14-1.58) for SBP values of $120-129 \mathrm{~mm} \mathrm{Hg}$, 130-139 mm Hg and 140-149 mm Hg, respectively, compared with SBP $<120 \mathrm{~mm} \mathrm{Hg}$. For total stroke mortality, the corresponding HRs were 1.29 (1.02-1.64), 1.37 (1.09-1.72) and 1.52 (1.20-1.93), while for IHD mortality, the corresponding HRs were 0.90 (0.64-1.26), $0.86(0.62-1.19)$ and $1.29(0.93-1.78)$, respectively.

Non-linear associations were significant for IHD.

Conclusions In the elderly Korean population, SBPs of 130-139 mm Hg elevated total stroke mortality, but not IHD mortality, compared with normal blood pressure, and a linear association was not observed for IHD mortality in the range $<140 \mathrm{~mm} \mathrm{Hg}$.
\end{abstract}

\section{INTRODUCTION}

Elevated blood pressure (BP) remains among the top causes of disease burden worldwide. ${ }^{12}$ The recent US guidelines for hypertension have lowered the diagnostic cut-off to $130 / 80 \mathrm{~mm} \mathrm{Hg}$, including the elderly population. ${ }^{3}$ However, uncertainties remain regarding the effect of $\mathrm{BP}$ on various cardiovascular outcomes in different age groups. Indeed, the relative risks for BP on cardiovascular outcomes decrease with age, ${ }^{2}$ and not all cardiovascular disease (CVD) subtypes exhibit linear relationships with $\mathrm{BP}^{4-7}$ Moreover, it is not clear whether a systolic blood pressure (SBP) of 130-139 mm Hg elevates risk compared with normal $\mathrm{BP}(\mathrm{SBP}<120 \mathrm{~mm} \mathrm{Hg})$ for each subtype of cardiovascular outcomes in the elderly. A detailed analysis of the age-specific effects of BP on various cardiovascular outcomes would help establish individualised treatment recommendations in this era of a rapidly ageing society.
Although several large-scale epidemiological studies have addressed the age-specific relationships between BP and cardiovascular outcomes, most studies included hypertensive patients already taking medications and were largely based on data from Western populations. ${ }^{8} 9$ To establish an effective preventive strategy in Asian populations, prospective observational studies reflecting Asian-specific characteristics are needed. However, limited and even conflicting reports exist in studies on elderly Asians. ${ }^{10-13}$ The recent Chinese Multi-provincial Cohort Study found that no risk elevation was associated with an SBP of 130-139 mm Hg compared with normal BP in those aged $\geq 60$ years. ${ }^{12}$ In the current study, we aimed to clarify whether SBP shows a linear association with cause-specific CVD mortality in all age groups and to identify whether an SBP of $130-139 \mathrm{~mm}$ $\mathrm{Hg}$ elevates CVD mortality in elderly individuals without known hypertension or CVD at baseline. For this analysis, we used a nationwide prospective cohort from Korea, and we confined the focus of our analysis to SBP, which has been reported to be a better predictor of cardiovascular outcomes than other BP parameters. ${ }^{8} 14$

\section{METHODS}

\section{Study population and follow-up}

The National Health Insurance Service (NHIS) provides compulsory health insurance that covers $97 \%$ of the Korean population. The study cohort $(\mathrm{n}=514795)$ was a $10 \%$ random sample of 5.15 million NHIS health screening participants during 2002-2003 who were aged 40-79 years in $2002 .{ }^{15}$ We excluded 85575 individuals due to missing information $(n=1595)$ on body mass index (BMI), serum fasting glucose, total cholesterol and SBP; known heart disease or stroke $(n=9695)$; or known prevalent hypertension $(n=74285)$ at baseline as assessed by the NHIS claim database. For the remaining 429220 people, follow-up for causes of death was carried out until 31 December 2013 through national death records using unique personal identification numbers. The International Classification of Diseases-10th Revision was used to identify cases of death from CVD (I00-I99), and the subtypes of CVD mortality were classified into ischaemic heart disease (IHD; I20-I25), acute myocardial infarction (MI; I21), total stroke (I60I69), haemorrhagic stroke (I60-I62), subarachnoid haemorrhage (SAH; I60), intracerebral haemorrhage (ICH; I61-I62) and ischaemic stroke (I63). In accordance with the conditions stipulated in Korean laws, health examination data can be provided for 
research without specific informed consent from the participants. Anonymised data were provided to the authors by NHIS.

\section{Data collection}

Weight and height were measured to the nearest kilogram and centimetre, respectively, while participants wore light clothing without shoes. BMI was calculated as weight in kilograms divided by the square of height in metres. BP was measured by a trained staff or a nurse in a seated position after resting for at least $5 \mathrm{~min}$. Fasting serum glucose was assayed using enzymatic methods. Smoking history, alcohol use, physical activity, and history of heart disease, stroke and cancer were reported via a questionnaire. Health examination and data collection followed a standard protocol officially documented by the Ministry of Health and Welfare. The external quality validation process for clinical chemistry in participating hospitals was supervised by the Korean Association of Quality Assurance for Clinical Laboratories, and quality assessment was performed regularly. We considered individuals to have prevalent hypertension at baseline if they visited a medical institution for hypertension (I10I15) at least once within 6 months before or 2 months after the baseline health examination date.

\section{Statistical analysis}

Baseline was defined as the time of the health examination. To identify age-specific effects, participants were categorised into three groups based on age at baseline (40-59 years, 60-69 years and $70-80$ years). SBP values were categorised into six categories ( $<120 \mathrm{~mm} \mathrm{Hg}$ (reference), $120-129 \mathrm{~mm} \mathrm{Hg}, 130-139 \mathrm{~mm} \mathrm{Hg}$, $140-149 \mathrm{~mm} \mathrm{Hg}, 150-159 \mathrm{~mm} \mathrm{Hg}$ and $\geq 160 \mathrm{~mm} \mathrm{Hg}$ ). SBP was also analysed as a continuous variable, assuming a linear association. Non-linear associations were evaluated using a restricted cubic spline transformation of SBP with three knots (predefined at the 5th, 50th and 95th centiles in each analysis).

The HRs and CIs for CVD deaths were calculated using Cox proportional hazards models in each age group, further stratified by age (years) at baseline (40-44 years, $45-54$ years, $55-64$ years, 65-74 years and 75-80 years) after adjusting for age at baseline (continuous variable; within each age group), sex, smoking status (current smoker, former smoker, never smoker and missing information), alcohol consumption (none, $<10 \mathrm{~g}$ ethanol/day, 10-39 g ethanol/day, $\geq 40 \mathrm{~g}$ ethanol/day and missing information), physical activity (at least once per week; yes and no), beneficiary income status (deciles; below 4 (low income), 4-7, 8-10 (high income)), BMI (continuous variable), total cholesterol (continuous variable) and fasting glucose (continuous variable). In the Cox models, the cause-specific hazard method was used for dealing with competing risks; individuals who experienced a competing event (other causes of death) or reached the end of the follow-up were treated as censored. ${ }^{16}$ Subgroup analyses were performed to examine differences in the associations according to age and sex. Cochran's Q statistic was used as the interaction test to examine the difference in the effect size of SBP between age groups. The proportional hazards assumption was examined by using Schoenfeld residuals. ${ }^{17}$ No evidence was found of violation of the proportional hazards assumption for each continuous and dummy variable of SBP for IHD mortality. A sensitivity analysis that excluded those who died within the first 3 years of follow-up was conducted, and subgroup analyses were also used for sensitivity testing. All p values were two-sided. All analyses were conducted using SAS V.9.4 software (SAS Institute, Cary, North Carolina, USA).

\section{RESULTS}

\section{Baseline demographic findings}

Among the 429220 participants, 237738 (55.4\%) were men. Their mean age was 51.8 (SD 9.3) years and their mean SBP was 124.7 (SD 16.9) mm Hg. Overall, 92572 (21.6\%) participants had an SBP $\geq 140 \mathrm{~mm} \mathrm{Hg}$ (table 1). Clinical profiles classified by SBP category are provided in online supplementary table 1 . During 10.4 years of follow-up, a total of 4319 cardiovascular deaths occurred.

\section{Associations between SBP and overall/individual CVD mortality in the overall population}

In the overall population, every $20 \mathrm{~mm} \mathrm{Hg}$ elevation in SBP was associated with a $40 \%$ elevated risk of overall cardiovascular death (HR 1.40 (1.36-1.44)). The association with total stroke mortality was stronger than the association with IHD mortality (HRs were $1.51(1.45-1.58)$ for total stroke and 1.31 (1.231.40) for IHD). For stroke subtypes, haemorrhagic stroke (HR 1.65 (1.49-1.57)) exhibited a stronger association with SBP than ischaemic stroke (HR 1.27 (1.11-1.45), online supplementary table 2).

\section{Age-specific associations of SBP with overall/individual CVD mortality}

When stratified by age, assuming a linear association, the strength of association weakened according to ageing ( $p$ for interaction $<0.001$ for overall CVD mortality), but remained significant even in the elderly group for various causes of CVD mortality (figure 1, online supplementary table 3). In detail, each $20 \mathrm{~mm} \mathrm{Hg}$ elevation in SBP was associated with 1.67-fold (95\% CI 1.57 to 1.78 ) elevated risk for overall CVD mortality in those aged $40-59$ years, 1.40 -fold increase $(95 \%$ CI 1.33 to 1.48 ) in those aged $60-69$ years and 1.22 -fold increase $(95 \% \mathrm{CI}$ 1.16 to 1.29 ) in those aged $70-80$ years.

In the categorical analyses, a positive and graded association was generally observed between SBP and both overall and cause-specific CVD mortality regardless of age, except for IHD mortality in those aged $70-80$ years (figure 2 , online supplementary tables 4 and 5). In persons aged 70-80 years, for overall CVD mortality, the HRs $(95 \% \mathrm{CI})$ were $1.08(0.92-1.28)$ for SBP of $120-129 \mathrm{~mm} \mathrm{Hg}, 1.14(0.97-1.34)$ for SBP of $130-139 \mathrm{~mm}$ $\mathrm{Hg}, 1.34$ (1.14-1.58) for SBP of 140-149 mm Hg, 1.56 (1.311.86) for SBP of $150-159 \mathrm{~mm} \mathrm{Hg}$ and $1.81(1.52-2.17)$ for $\mathrm{SBP} \geq 160 \mathrm{~mm} \mathrm{Hg}$ when compared with SBP $<120 \mathrm{~mm} \mathrm{Hg}$. For IHD mortality, the corresponding HRs $(95 \% \mathrm{CI})$ were 0.90 (0.64-1.26), 0.86 (0.62-1.19), 1.29 (0.93-1.78), 1.44 (1.02$2.04)$ and 1.58 (1.11-2.27), respectively. For acute MI, a similar pattern was observed.

The subgroup analysis by sex revealed similar results to those of the main analysis (online supplementary tables 6 and 7 and supplementary figures 1 and 2). The sensitivity analysis excluding those who died within the first 3 years of follow-up also yielded similar results (online supplementary table 8 and figure 3).

When the shape of the association was explored using a restricted cubic spline model (figure 3), the results were similar to those obtained from the categorical analysis. The slope of the association was steeper for mortality from stroke than for mortality from IHD in each age group, and the relationship was generally stronger in the younger population. Non-linear associations were significant for IHD (including acute MI) mortality in people aged 70-80 years. The risk for IHD (including acute MI) mortality was lowest around an SBP of $120-130 \mathrm{~mm} \mathrm{Hg}$ in the elderly. 


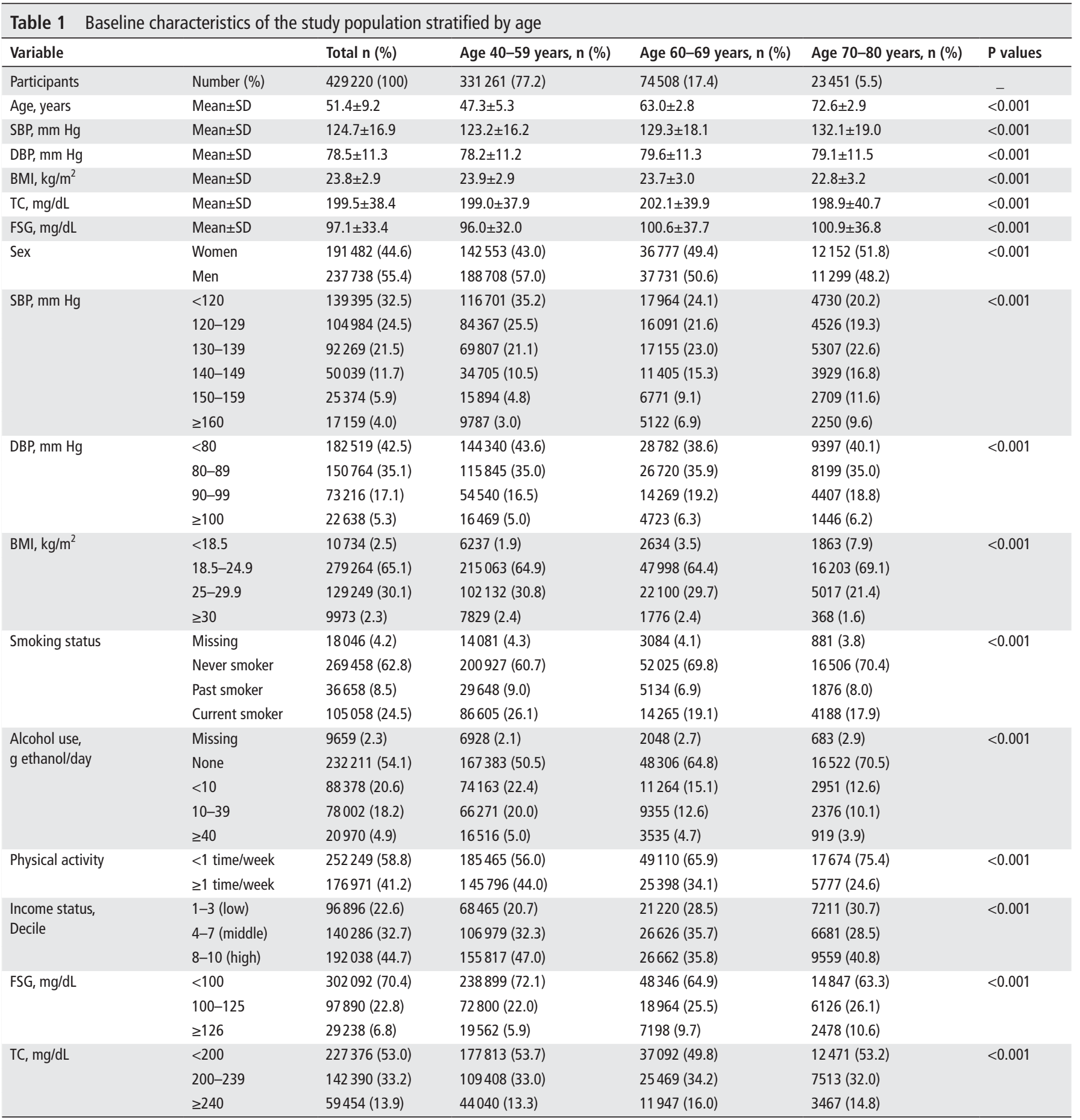

*83 missing values existed for diastolic blood pressure.

BMI, body mass index; DBP, diastolic blood pressure; FSG, fasting serum glucose; SBP, systolic blood pressure; TC, total cholesterol.

\section{DISCUSSION}

\section{Association of SBP with CVD mortality: a focus on the age- specific effect}

Currently, the suggested thresholds for hypertension in the elderly differ between the European (SBP $\geq 140 / 90 \mathrm{~mm} \mathrm{Hg}$ ) and US (SBP $\geq 130 \mathrm{~mm} \mathrm{Hg}$ ) guidelines. ${ }^{3}{ }^{18}$ Unfortunately, scant data support the establishment of an appropriate cut-off for hypertension in Asian populations. In this prospective cohort involving over 0.4 million Korean adults who initially had no known hypertension or CVD, we found that, unlike other age groups, those aged 70-80 years demonstrated a non-linear association between SBP and IHD mortality. An SBP of $130-139 \mathrm{~mm} \mathrm{Hg}$ was not associated with increased IHD mortality compared with normal BP (SBP $<120 \mathrm{~mm} \mathrm{Hg}$ ). Although an SBP of 130-139 mm $\mathrm{Hg}$ was associated with elevated stroke mortality, the overall CVD mortality for an SBP of 130-139 mm Hg increased by only $14 \%$. Furthermore, the association was non-significant in those aged 70-80 years, casting doubt on the appropriateness of incorporating an SBP of 130-139 mm Hg into the definition of hypertension in elderly Koreans.

Regarding the effect of BP on CVD outcomes in the elderly without a previous history of CVD, there have been limited and conflicting results. ${ }^{4}{ }^{8-10} 13$ In the current analysis, a reverse-L-shaped association, with risk elevation beginning at 
Aged 40-59 yrs

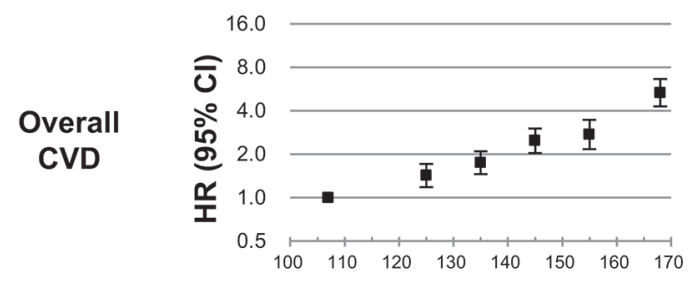

IHD

Acute MI



\section{$\widehat{\bar{U}}$}

บำ

ํํㅇ 2
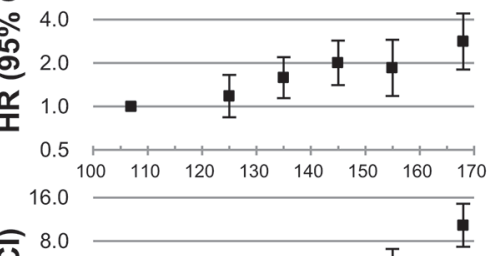

Total stroke

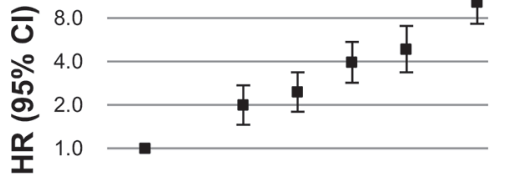

Haemorrhagic
Stroke

$\overline{0} 8.0$


呈 1.0


ত
웅
옹
또

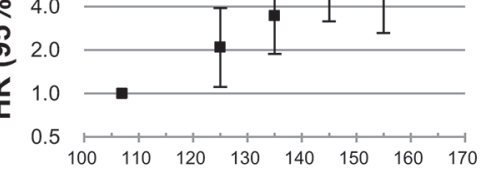

ICH
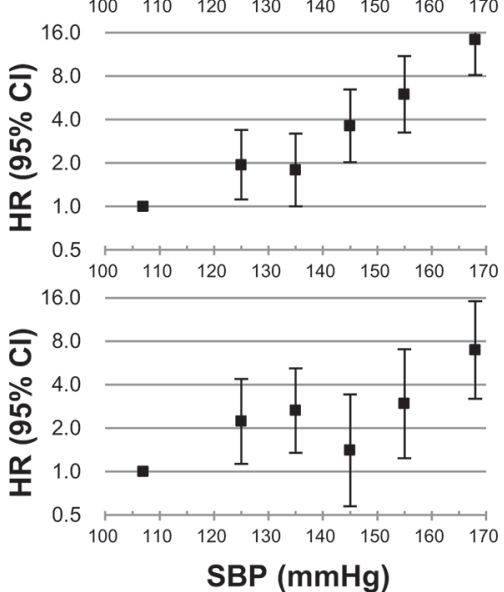

Aged 60-69 yrs

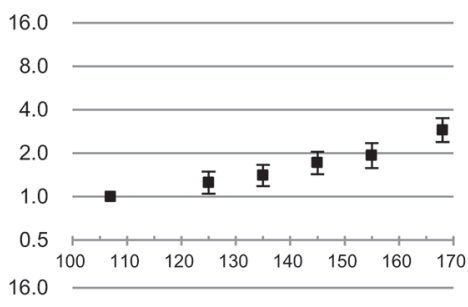

8.0

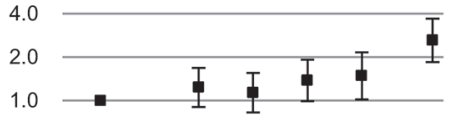

0.5

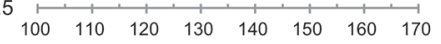

16.0

8.0

2.0

1.0

16.0

100

8.0


1.0
0.5
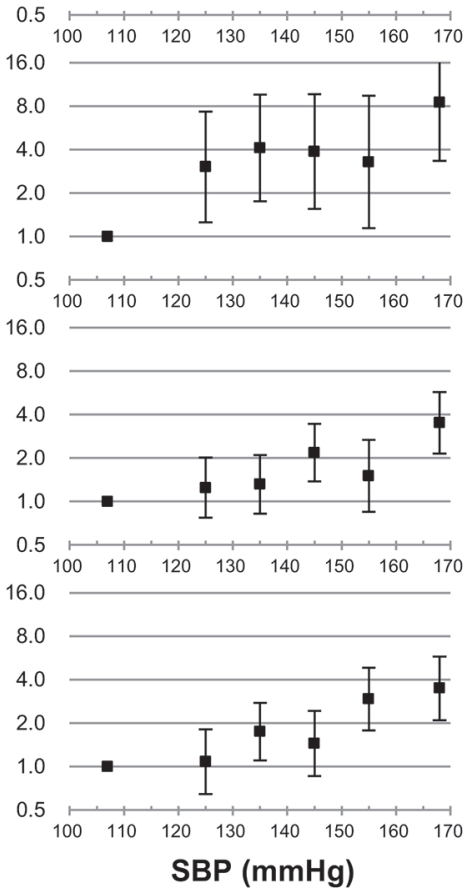

Aged $70-80$ yrs

16.0

4.0

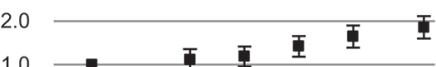

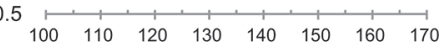

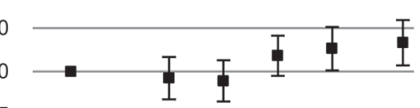

$\begin{array}{llllllll}100 & 110 & 120 & 130 & 140 & 150 & 160 & 170\end{array}$

16.0

8.0

2.0

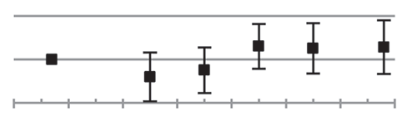

$\begin{array}{llllllll}100 & 110 & 120 & 130 & 140 & 150 & 160 & 170\end{array}$

160

8.0

4.0
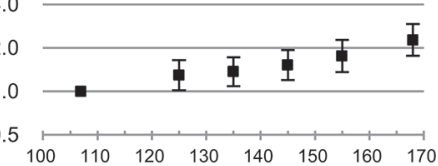

6.0

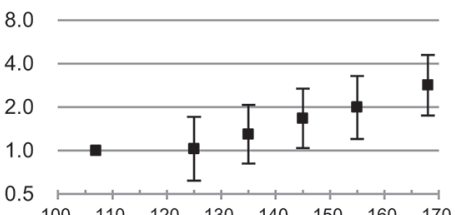


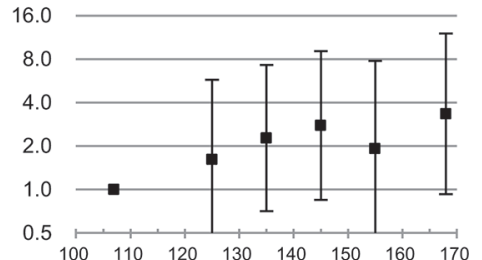

16.0


SBP $(\mathrm{mmHg})$

Figure 2 Risk for CVD mortality by SBP category in the overall population, stratified by age. CVD, cardiovascular disease; ICH, intracranial haemorrhage; IHD, ischaemic heart disease; MI, myocardial infarction; SAH, subarachnoid haemorrhage; SBP, systolic blood pressure. 



Figure 3 Shape of the association between SBP and CVD mortality using a restricted cubic spline model. CVD, cardiovascular disease; IHD, ischaemic heart disease; MI, myocardial infarction; SBP, systolic blood pressure.

outcomes, which has been found to be especially prominent in the elderly. ${ }^{4-7} 13$ 19-24 The possibility of reverse causality may also be considered. However, we rigorously excluded patients with known CVD, and the participants in the current study represented a relatively healthy population capable of visiting a clinic. Furthermore, similar results were obtained after excluding subjects who died within the first 3 years of follow-up.

\section{Other findings}

Similar to previous studies, at younger age, the strength of the association between SBP and cause-specific CVD mortality was stronger, ${ }^{2} 89$ and an SBP of $130-139 \mathrm{~mm} \mathrm{Hg}$ was associated with increased overall and individual CVD mortality. ${ }^{12} 25$ Additional interventional studies are needed to clarify the effect of early management of an SBP of $130-139 \mathrm{~mm} \mathrm{Hg}$ in the young and middle-aged population in light of cardiovascular benefits, as well as cost-effectiveness and long-term medication use. ${ }^{26}$ Furthermore, our data showed the stroke-prone characteristics of Asian populations, as BP showed a stronger association with stroke than with IHD. ${ }^{10} 11$ Among stroke subtypes, haemorrhagic stroke showed a steeper slope of association than ischaemic stroke, similar to the findings of a study in the China 
Kadoorie Biobank population. ${ }^{11}$ However, the study of Lacey et al did not distinguish between different subtypes of haemorrhagic stroke. Currently, limited data are available regarding the effects of BP on SAH. In our study, the relationship of SBP with SAH mortality was generally similar among different age groups. Conversely, in the study by Rapsomaniki et al, the pattern of association between SBP and SAH was different in the elderly. ${ }^{9}$ It is also noteworthy that the steeper slope of the association for haemorrhagic stroke than for ischaemic stroke was more evident in elderly adults (aged 70-80 years).

\section{Clinical implications}

SBP is a vital CVD risk factor regardless of age; meanwhile, our research indicates that the 'lower is better' concept might not be directly applicable to elderly subjects with an SBP of $130-139 \mathrm{~mm} \mathrm{Hg}$, particularly for IHD mortality prevention. Furthermore, the number needed to treat to prevent one death per year from overall CVD, calculated by inverting the age-sex adjusted mortality difference, was high (2118) for a baseline SBP of 130-139 mm Hg and the proportion of overall CVD deaths attributable to SBP $130-139 \mathrm{~mm} \mathrm{Hg}$ was $2.5 \%$ (95\% CI $0.4 \%$ to $5.4 \%$ ) in those aged $70-80$ years (online supplementary table 9). This observation raises the concern that it may be ineffective to define an SBP of 130-139 $\mathrm{mm} \mathrm{Hg}$ as hypertension in elderly individuals with a relatively low risk of CVD from a cost-effectiveness perspective.

\section{Strengths and limitations}

The current study was a population-based study involving a large number of participants and substantial and complete follow-up, which enabled a thorough evaluation of the age-specific effects of SBP on cause-specific CVD. Our study could serve as a useful reference for identifying SBP-related risks in the general population. Further, the current study provided a detailed classification of the subtypes of stroke (ischaemic, ICH and SAH). Lastly, our results remained robust even after extensively controlling for possible confounders.

Several limitations in the present study should be noted. First, the BP measurements could have been less than ideal, because health examination was performed in various centres and BP was evaluated only once at baseline. Thus, the relative risks associated with SBP might have been underestimated due to non-differential misclassification of $\mathrm{BP}$ and the regression dilution effect. ${ }^{27}$ Second, the cause of death on Korean death certificates might have been subject to misclassification. However, death certificates have been found to be reasonably valid in comparison with medical records. ${ }^{28}$ In patients with stroke, CT and MRI are routinely performed $(>90 \%)$ in Korea. ${ }^{29}$ Third, we examined CVD mortality, but not non-fatal events. The associations between BP and mortality might have been different from those between BP and the incidence of non-fatal events. Fourth, our sample exclusively comprised Koreans, limiting the generalisability of our findings to other ethnicities. Lastly, the nature of an observational study limits causal inferences, and our findings therefore cannot directly indicate the most appropriate BP cut-off for initiating BP interventions. However, it could serve as a basis for designing future clinical trials or for setting individualised treatment strategies in the elderly.

\section{CONCLUSIONS}

In a Korean general population without a previous history of hypertension or CVD, we observed a non-linear relationship between SBP and IHD mortality in the elderly, while we demonstrated that elevated BP is a significant risk factor across all age groups. Furthermore, elderly adults with an SBP of $130-139 \mathrm{~mm} \mathrm{Hg}$ did not show a significant elevation in mortality from IHD or overall CVD. Given the rapidly growing ageing population, subsequent interventional and/or epidemiological studies would be helpful to clarify whether the initiation of BP interventions at a lower threshold would lead to improvements in various cardiovascular outcomes across age and racial groups.

\section{Key messages}

What is already known on this subject?

- Elevated blood pressure (BP) is a well-known risk factor and the topmost disease burden worldwide. Recent US guidelines lowered the diagnostic threshold for hypertension to $130 / 80 \mathrm{~mm} \mathrm{Hg}$ in the elderly population. There is a concern that people with a systolic blood pressure (SBP) of 130$139 \mathrm{~mm} \mathrm{Hg}$ can become mandated for BP intervention.

\section{What might this study add?}

- Although the present study generally supports the idea of SBP as a vital cardiovascular risk factor regardless of age, the elderly population (those aged $70-80$ years) demonstrated a non-linear association between SBP and ischaemic heart disease (IHD) mortality. Furthermore, elderly adults with an SBP of $130-139 \mathrm{~mm} \mathrm{Hg}$ did not show a significant elevation in mortality for IHD or overall cardiovascular disease.

\section{How might this impact on clinical practice?}

- An individualised approach considering each patient's personal risk profile might be needed when initiating BP interventions for the elderly. Given the rapidly growing ageing population, subsequent interventional or epidemiological studies would be helpful to clarify whether the initiation of BP interventions at a lower threshold would lead to improvements in various cardiovascular outcomes across age and racial groups.

Acknowledgements The authors thank the staff at the Big Data Steering Department at the NHIS for providing the data and support.

Contributors $\mathrm{M}-\mathrm{HJ}$ and S-WY conceived and designed the study. S-WY acquired data and performed statistical analysis. M-HJ and S-WY wrote the first draft. M-HJ, S-WY, SJA, and J-JY analysed and interpreted data and contributed to critical revision of the manuscript. All authors have read and approved of the final submitted version of the manuscript. S-WY is the study guarantor.

Funding The authors have not declared a specific grant for this research from any funding agency in the public, commercial or not-for-profit sectors.

Competing interests None declared.

Patient consent for publication Not required.

Ethics approval This study was approved by the Institutional Review Board of Catholic Kwandong University, Republic of Korea.

Provenance and peer review Not commissioned; externally peer reviewed.

Data sharing statement The data are available from the NHIS of Korea (http:// nhiss.nhis.or.kr/bd/ab/bdaba000eng.do). Applicants to use the data should contact the NHIS (Office of big data operation, +82-33-736-2469) for further information.

\section{REFERENCES}

1 GBD. Risk Factors Collaborators. Global, regional, and national comparative risk assessment of 79 behavioural, environmental and occupational, and metabolic risks or clusters of risks, 1990-2015: a systematic analysis for the Global Burden of Disease Study 2015. Lancet 2015;2016:1659-724.

2 Rahimi K, Emdin CA, MacMahon S. The epidemiology of blood pressure and its worldwide management. Circ Res 2015;116:925-36. 
3 Whelton PK, Carey RM, Aronow WS, et al. ACC/AHA/AAPA/ABC/ACPM/AGS/APhA/ ASH/ASPC/NMA/PCNA Guideline for the Prevention, Detection, Evaluation, and Management of High Blood Pressure in Adults: A Report of the American College of Cardiology/American Heart Association Task Force on Clinical Practice Guidelines. J Am Coll Cardiol 2017;2018:e127-248.

4 Yi SW, Mok Y, Ohrr H, et al. Low systolic blood pressure and vascular mortality among more than 1 million korean adults. Circulation 2016:133:2381-90.

5 Bergmark BA, Scirica BM, Steg PG, et al. Blood pressure and cardiovascular outcomes in patients with diabetes and high cardiovascular risk. Eur Heart J 2018;39:2255-62.

6 Böhm M, Schumacher H, Teo KK, et al. Achieved blood pressure and cardiovascular outcomes in high-risk patients: results from ONTARGET and TRANSCEND trials. Lancet 2017;389:2226-37.

7 Kannel WB, D'Agostino RB, Silbershatz H. Blood pressure and cardiovascular morbidity and mortality rates in the elderly. Am Heart J 1997;134:758-63.

8 Lewington S, Clarke R, Qizilbash N, et al. Age-specific relevance of usual blood pressure to vascular mortality: a meta-analysis of individual data for one million adults in 61 prospective studies. Lancet 2002;360:1903-13.

9 Rapsomaniki E, Timmis A, George J, et al. Blood pressure and incidence of twelve cardiovascular diseases: lifetime risks, healthy life-years lost, and age-specific associations in 1.25 million people. Lancet 2014;383:1899-911.

10 Lawes CM, Rodgers A, Bennett DA, et al. Asia Pacific Cohort Studies Collaboration. Blood pressure and cardiovascular disease in the Asia Pacific region. J Hypertens 2003;21:707-16.

11 Lacey B, Lewington S, Clarke R, et al. Age-specific association between blood pressure and vascular and non-vascular chronic diseases in 0.5 million adults in China: a prospective cohort study. Lancet Glob Health 2018;6:e641-e649.

12 Qi Y, Han X, Zhao D, et al. Long-term cardiovascular risk associated with stage 1 hypertension defined by the 2017 acc/aha hypertension guideline. J Am Coll Cardiol 2018;72:1201-10.

13 Arima H, Tanizaki Y, Kiyohara Y, et al. Validity of the JNC VI recommendations for the management of hypertension in a general population of Japanese elderly: the Hisayama study. Arch Intern Med 2003;163:361-6.

14 Franklin SS, Lopez VA, Wong ND, et al. Single versus combined blood pressure components and risk for cardiovascular disease: the Framingham Heart Study. Circulation 2009;119:243-50.

15 Seong SC, Kim YY, Park SK, et al. Cohort profile: the National Health Insurance Service-National Health Screening Cohort (NHIS-HEALS) in Korea. BMJ Open 2017;7:e016640.
16 Lau B, Cole SR, Gange SJ. Competing risk regression models for epidemiologic data. Am J Epidemiol 2009;170:244-56.

17 Allison PD. Survival analysis using SAS: a practical guide. 2nd edition. North Carolina, 2010.

18 Williams B, Mancia G, Spiering W, et al. 2018 Practice Guidelines for the management of arterial hypertension of the European Society of Hypertension and the European Society of Cardiology: ESH/ESC Task Force for the Management of Arterial Hypertension. J Hypertens 2018;36:2284-309.

19 JATOS Study Group. Principal results of the Japanese trial to assess optimal systolic blood pressure in elderly hypertensive patients (JATOS). Hypertens Res 2008;31:2115-27.

20 Ogihara T, Saruta T, Rakugi H, et al. for the Valsartan in Elderly Isolated Systolic Hypertension Study Group. Target blood pressure for treatment of isolated systolic hypertension in the elderly: Valsartan in Elderly Isolated Systolic Hypertension Study. Hypertension 2010;56:196-202.

21 Messerli FH, Panjrath GS. The J-curve between blood pressure and coronary artery disease or essential hypertension: exactly how essential? J Am Coll Cardiol 2009;54:1827-34.

22 Staessen JA, Gasowski J, Wang JG, et al. Risks of untreated and treated isolated systolic hypertension in the elderly: meta-analysis of outcome trials. Lancet 2000;355:865-72.

23 Mancia G, Kjeldsen SE, Zappe DH, et al. Cardiovascular outcomes at different ontreatment blood pressures in the hypertensive patients of the VALUE trial. Eur Heart J 2016:37:955-64.

24 Bangalore S, Messerli FH, Wun CC, et al. Treating to New Targets Steering Committee and Investigators. J-curve revisited: An analysis of blood pressure and cardiovascular events in the Treating to New Targets (TNT) Trial. Eur Heart J 2010;31:2897-908.

25 Son JS, Choi S, Kim K, et al. Association of blood pressure classification in korean young adults according to the 2017 american college of cardiology/american heart association guidelines with subsequent cardiovascular disease events. JAMA 2018;320:1783-92.

26 Sniderman AD, Toth PP, Thanassoulis $G$, et al. Taking a longer term view of cardiovascular risk: the causal exposure paradigm. BMJ 2014;348:g3047.

27 Clarke R, Shipley M, Lewington S, et al. Underestimation of risk associations due to regression dilution in long-term follow-up of prospective studies. Am J Epidemiol 1999;150:341-53.

28 Won TY, Kang BS, Th I, et al. The study of accuracy of death statistics. J Korean Soc Emerg Med 2007;18:256-62.

29 Ministry of Health and Welfare. Pilot test of National Cardiovascular Disease Surveillance System. Seoul, Korea: Ministry of Health and Welfare, 2000. 